\title{
Heterogeneity in Cystic Echinococcosis Management Among Spanish Centers: Results from a National Survey
}

\author{
Fernando Salvador, ${ }^{1 *}$ Eva Calabuig, ${ }^{2}$ Rogelio López-Vélez, ${ }^{3}$ Javier Pardo-Lledías, ${ }^{4}$ Diego Torrús, ${ }^{5}$ María Peñaranda, ${ }^{6}$ \\ Azucena Rodríguez-Guardado, ${ }^{7}$ Milagros García López-Hortelano, ${ }^{8}$ and Moncef Belhassen-García ${ }^{9}$ for the Hydatidosis Working \\ Group of the Spanish Society of Tropical Medicine and International Health (SEMTSI) \\ ${ }^{1}$ Department of Infectious Diseases, Vall d'Hebron University Hospital, Universitat Autònoma de Barcelona, PROSICS Barcelona, Barcelona, \\ Spain; ${ }^{2}$ Infectious Diseases Unit, La Fe University and Polytechnic Hospital, University of Valencia, Valencia, Spain; ${ }^{3}$ nfectious Diseases \\ Department, National Referral Unit for Tropical Diseases, Ramón y Cajal University Hospital, Madrid, Spain; ${ }^{4}$ Department of Internal Medicine, \\ Hospital Universitario Marqués de Valdecilla, Universidad de Cantabria, IDIVAL, Santander, Spain; 5 Infectious Diseases Unit, Hospital General \\ Universitario de Alicante, Alicante, Spain; ${ }^{6}$ Department of Internal Medicine, Son Espases University Hospital, Palma de Mallorca, Spain; \\ ${ }^{7}$ Department of Internal Mecicine, Hospital Universitario de Cabueñes, Gijón, Spain; ${ }^{8}$ Infectious Diseases Department, La Paz-Carlos III Pediatric \\ University Hospital, Madrid, Spain; ${ }^{9}$ Department of Internal Medicine, Infectious Diseases Section, Complejo Asistencial Universitario de \\ Salamanca, Salamanca, Spain
}

\begin{abstract}
The aim of the study was to evaluate the availability of different procedures, diagnostic tests, and treatments, as well as the procedures and techniques used in the management of cystic echinococcosis (CE) in Spain. This was a cross-sectional study performed from September to December 2018 in Spain. A survey directed to CE-treating clinicians was conducted to collect information regarding the center characteristics and the different protocols of management followed. Thirty-nine centers among 76 contacted centers participated in the survey, most of them belonging to the public health system and attending both adult and children. The median number of patients with CE attended during the last three years per center was 15. Percutaneous techniques were used only in seven centers, and surgery was the most frequently used therapeutic approach. Drugs and duration of treatment (both when administered exclusively or when combined with surgery/puncture, aspiration, injection, and reaspiration) were very variable depending on the centers. There is a high variability in the management of CE among Spanish centers. These results stress the importance of promoting the diffusion of existing knowledge, adapting the WHO recommendations to our setting, and referring patients to referral centers at a national level.
\end{abstract}

\section{INTRODUCTION}

Cystic echinococcosis (CE) is a zoonotic disease caused by the larval stages of the cestode Echinococcus granulosus and is one of the 20 neglected tropical diseases listed by the WHO. ${ }^{1}$ Although CE is distributed worldwide, most cases are described in South America, Africa, and Asia. In Europe, the countries with higher prevalence are those placed in the Mediterranean Basin (such as Portugal, Spain, Italy, and Greece), where annual incidence rates for human CE of 4-8 per 100,000 inhabitants have been reported. ${ }^{2}$ In Spain, although there was a decrease in the incidence during the last decades, human CE is still one of the most important anthropozoonoses in terms of morbidity and economic losses. $^{3}$

The current diagnostic criteria for CE rely on the expert consensus for the CE diagnosis performed by the WHO Informal Working Group on Echinococcosis and published in 2010. These diagnostic criteria include clinical, epidemiological, radiological, serological, and histopathological variables. ${ }^{4}$ Regarding the treatment of $\mathrm{CE}$, there are four main options available: surgery, antiparasitic treatment (mainly with benzimidazoles and praziquantel), percutaneous techniques (such as puncture, aspiration, injection, and reaspiration (PAIR) or modified catheter treatment), and the "watch and wait" strategy. Despite the WHO recommendations, there is a lack of standardization that leads to a high variability in the management of this pathology, as it has been evidenced in a *Address correspondence to Fernando Salvador, Department of
Infectious Diseases, Vall d'Hebron University Hospital, $P^{\circ}$ Vall
d'Hebron 119-129, Barcelona 08035, Spain. E-mail: fmsalvad@ vhebron.net recent survey asking 41 specialists about their proposed management of five CE clinical cases. ${ }^{5}$

The Hydatidosis Working Spanish Group (HWSG) of the Spanish Society of Tropical Medicine and International Health (SEMTSI) was created in 2018 with the aim of gathering experts of different disciplines (infectious diseases, microbiology, radiology, and surgery) to elaborate a consensus document for the diagnosis and treatment of $C E$ at a national level. As a secondary objective, the HWSG of the SEMTSI aimed to evaluate the availability of different procedures, diagnostic tests, and treatments, as well as the use of these techniques and procedures in the management of CE around the country, which was the objective of the present study.

\section{METHODS}

This is a cross-sectional study performed by the HWSG of the SEMTSI from September to December 2018. A survey directed to CE-treating clinicians was conducted to collect information regarding the center characteristics and the different protocols of CE management followed in each center. Survey recipients (one per center) were identified in three ways: members of the HWSG, clinicians of other centers contacted through the members of the HWSG, and through a PubMed search to identify Spanish clinicians who had published articles on CE. After the identification of the potential recipients of the survey, they were sent e-mails explaining the nature and objectives of the study, and the survey was sent to them when accepting to participate. When the information of the survey was incomplete or confusing, participants were contacted to clarify the information.

The survey was elaborated by the HWSG members and included general characteristics of the center (location, type of 
population attended, and level of specialization), general information regarding CE cases attended from 2015 to 2017 (number of total CE cases, the treatment they received, percentage of extrahepatic cases, and percentage of immigrant population among CE cases), available diagnostic and treatment options, and protocols followed by the centers. The survey is available in the Supplemental Material.

Categorical data were presented as absolute numbers and proportions, and continuous variables were expressed as medians and interquartile ranges (IQRs). The $x^{2}$ test was used to compare the distribution of categorical variables and the Mann-Whitney $U$ test for continuous variables. Results were considered statistically significant if the two-tailed $P$-value was $<0.05$. SPSS software for Windows (version 19.0; SPSS Inc, Chicago, IL) was used for statistical analyses. The Ethical Review Board of the Vall d'Hebron University Hospital (Barcelona, Spain) was consulted and agreed that ethical committee approval was not necessary. Procedures were performed in accordance with the ethical standards laid down in the Declaration of Helsinki as revised in 2013.

\section{RESULTS}

Seventy-six CE-treating clinicians were contacted, 48 accepted to participate in the study, and finally 39 sent back the filled survey. The geographical distribution of the participant centers was as follows (12 autonomous communities): Madrid (nine participants); Catalonia and Castilla y León (six participants each); Valencian Community (four centers); Balearic Islands, Andalucía, and Galicia (three centers each); Asturias, Aragón, Cantabria, Basque Country, and Canary Islands (one center each). Most of the centers belonged to the public health system; only two (5.1\%) centers had private activity. Fourteen (35.9\%) centers attended only adult patients, one $(2.6 \%)$ center attended only children (aged $<16$ years), and 24 $(61.5 \%)$ centers attended both adults and children. The median number of CE patients visited per center during the last 3 years was 15 (IQR: 5.75-24.25), and 25 (64.1\%) centers were visited by more than 15 patients. Characteristics of the centers and patients attended are summarized in Table 1.

Regarding the availability of different procedures and drugs, the following information was collected. Albendazole was

TABLE 1

Characteristics of the participant centers and patients attended

\begin{tabular}{lc}
\hline \multicolumn{1}{c}{ Characteristics and patients } & Number of centers $(n=39)$ \\
\hline Type of activity & \\
Public health system & $37 / 39(94.9 \%)$ \\
Private activity & $2 / 39(5.1 \%)$ \\
Population attended & $14 / 39(35.9 \%)$ \\
Only adults & $1 / 39(2.6 \%)$ \\
Only children & $24 / 39(61.5 \%)$ \\
Adults and children & \\
Number of patients attended in the last 3 & $15($ IQR $5.75-24.25)$ \\
years & $14 / 39(35.9 \%)$ \\
$\quad$ Median & $16 / 39(41 \%)$ \\
$\quad<10$ patients & $9 / 39(23.1 \%)$ \\
10-29 patients & $15 \%($ IQR $1.5-33 \%)$ \\
Z 30 patients & $26.5 \%($ IQR $10-61.5 \%)$ \\
Median percentage of extrahepatic cases & \\
Median percentage of cases in immigrant & \\
population & \\
\hline IQR $=$ interquartile range. Data are reported as number (\%) of centers or median (IQR).
\end{tabular}

available in 38 (97.4\%) centers, mebendazole in $34(87.2 \%)$ centers, and praziquantel in $33(84.6 \%)$ centers. Only 18 $(46.2 \%)$ centers had access to the percutaneous techniques. Five $(12.8 \%)$ centers had a multidisciplinary team, a preset group of infectious diseases specialists, surgeons, radiologists, and microbiologists, that regularly meets to discuss all CE cases.

Regarding the treatment option, the median percentage of each of them was $43.75 \%$ (IQR: $28.5-75 \%$ ) for surgery, $23.8 \%$ (IQR: 0-33.3\%) for "watch and wait," 15\% (IQR: 0-33.3\%) for exclusively pharmacological treatment, and 0\% (IQR: 0-0\%) for percutaneous techniques. Figure 1 shows the percentage of treatment option for each center. More information on the drugs used and duration of treatment is summarized in Table 2. Regarding the protocols used in each center, 14 (35.9\%) centers followed the WHO guidelines, two $(5.1 \%)$ centers followed their own institutional protocols, two $(5.1 \%)$ centers used other protocols, and 21 (53.8\%) did not follow any protocol or guideline.

Different medical and surgical departments diagnose and manage patients with CE depending on the center: department of surgery in 31 (79.5\%) centers, department of infectious diseases or tropical medicine unit in $28(71.8 \%)$ centers, department of internal medicine in 15 (38.5\%) centers, department of gastroenterology in nine (23.1\%) centers, and department of pneumology in three $(7.7 \%)$ centers. Diagnostic tools used for the patients' follow-up were as follows: ultrasounds used in $31(79.5 \%)$ centers, computed tomography in 30 (76.9\%) centers, serological tests in $25(64.1 \%)$ centers, and other techniques (magnetic resonance and positron emission tomography) in four (10.3\%) centers. Serological tests were available in 37 (94.9\%) centers, 21 (53.8\%) centers had an indirect hemagglutination test, 15 (38.5\%) had ELISA, six (15.4\%) had a chemiluminescence immunoassay, and two $(5.1 \%)$ had an indirect immunofluorescence test.

We compared centers by the number of patients visited in the last three years. Centers that attended more than 15 patients had higher access to percutaneous techniques $(60 \%$ versus $21.4 \%, P=0.020)$ and lower percentage of immigrant patients $(15 \%$ versus $61 \%, P=0.045)$ than the centers that attended 15 or less patients. No other differences were found. More information is summarized in Table 3.

\section{DISCUSSION}

Here, we present the results of a survey addressed to CEtreating clinicians from 39 Spanish centers (from 12 autonomous communities of 17). The information collected shows high variation regarding the availability of the different procedures and techniques depending on the centers, as well as heterogeneity in the therapeutic approach and recommendations/ guidelines followed, with different duration of pharmacological treatments and diverse procedures used (percutaneous techniques and surgery).

The number of patients attended in the last 3 years in each participant center was very variable and ranged from one patient to 44 patients. Centers attending larger number of patients were those with higher level of specialization or those placed in areas where CE incidence is higher. ${ }^{3}$ It could be argued that the variability among centers is related to the number of patients attended; however, when comparing centers by the number of patients attended, no relevant 


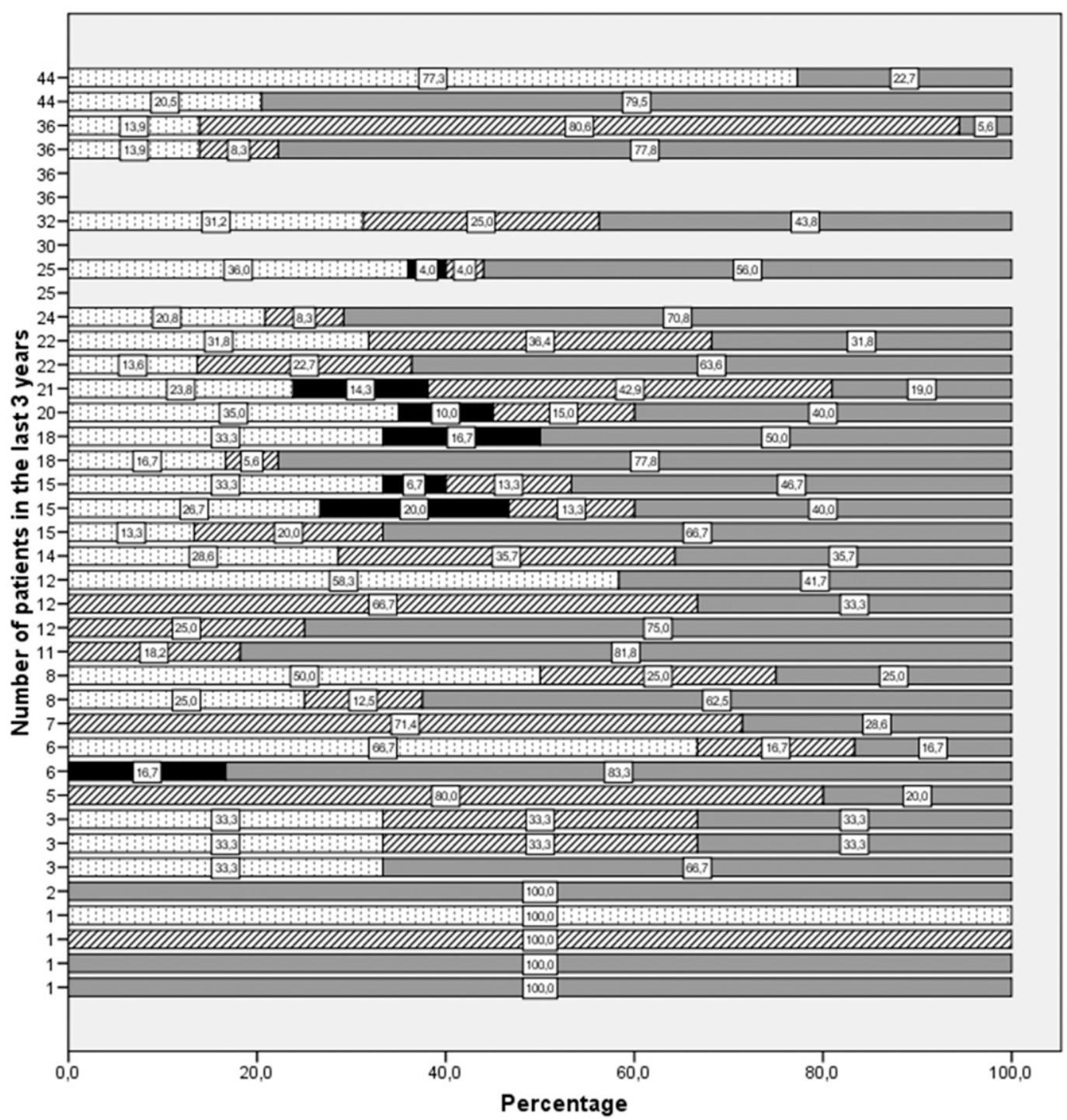

$\square$ SURGERY

Ø PHARMACOLOGICAL

PERCUTANEOUS TECHNIQUES

WATCH AND WAIT

Figure 1. Percentage of the different treatment options performed on patients attended in the last 3 years in each participant center.

differences were found. Hence, the variability seems to be unrelated to the number of patients or the level of specialization of the center.

One interesting finding of the survey was the high percentage of immigrant population among the CE cases reported by the centers (a median of $26.5 \%$ among centers). Scarce information is available regarding CE cases in immigrant population in Spain. Studies reporting Spanish case series of CE have described percentages of immigrant population ranging from $3 \%$ to $14 \% .^{6,7}$ Since 2015 , CE is a mandatory declaration disease in Spain. Moreover, veterinary control programs have been implemented in highest prevalence Spanish regions in the last decades. ${ }^{8}$ The changing epidemiological profile of CE diagnosed in Spain could be due to better disease control strategies in our country and the decrease of autochthonous cases, increasing the percentage of imported cases. Similarly, in Italy, cases in immigrant population are increasingly being diagnosed; in a recent study performed in Lombardy, $43 \%$ of the CE cases diagnosed in a single center were immigrants. ${ }^{9}$ Moreover, foreign cases diagnosed in our country may raise awareness of the incidence in the native countries of these patients, where the weakness of notification systems could underestimate the real burden of the disease.

Although the percutaneous techniques were available in almost half of the centers, only seven (18\%) centers used this technique during the last 3 years. The PAIR and other percutaneous techniques are recommended as an alternative to surgery for inoperable patients and those who refuse surgery and can be the first treatment option in cases of relapse after surgery or pharmacological treatment, especially in those within 5- to $10-\mathrm{cm} \mathrm{CE1} \mathrm{and} \mathrm{CE3a} \mathrm{cysts.}{ }^{4}$ In our study, even in centers with the highest number of patients attended, percutaneous techniques were not used. This technique, although being minimally invasive, needs well-trained interventional radiologists. In a recent study performed in a high-endemic area of Spain, only one patient from 491 (0.2\%) was treated with the PAIR technique. ${ }^{10}$ This fact should emphasize the importance of having well-trained personnel in these techniques; otherwise, the recommendations and guidelines are hard to follow in most of the centers.

Availability of different drugs used in CE treatment was quite high for each drug, albendazole being the most available drug. 
TABLE 2

Drugs used in the participant centers and duration of treatment

\begin{tabular}{lc}
\hline \multicolumn{1}{c}{ Drugs and duration of treatment } & Number of centers $(n=39)$ \\
\hline Number of centers using each drug & \\
Albendazole & $37 / 39(94.9 \%)$ \\
Praziquantel & $10 / 39(25.6 \%)$ \\
Mebendazole & $3 / 39(7.7 \%)$ \\
Albendazole + praziquantel & $17 / 39(43.6 \%)$ \\
Duration of treatment (pharmacological exclusively) & $3($ IQR $3-6)$ \\
Median (months) & $4 / 39(10.2 \%)$ \\
$<3$ months & $29 / 39(74.4 \%)$ \\
$3-6$ months & $6 / 39(15.4 \%)$ \\
$\geq 6$ months & $7($ IQR $7-30)$ \\
Duration of treatment before PAIR/ & \\
surgery (days) & $30($ IQR 28-60) \\
Duration of treatment after PAIR/surgery & \\
(days) & $19 / 39(48.7 \%)$ \\
Number of centers with patients treated & \\
with permanent pharmacological & \\
treatment & \\
\hline IQR = interquartile range; PAIR = puncture, aspiration, injection, and reaspiration. Data are \\
reported as number (\%) of centers or median (IQR).
\end{tabular}

However, the use of diverse drugs was very different depending on the centers. Surprisingly, there are centers still using mebendazole or praziquantel as a single drug, despite albendazole being a much more effective drug. The current evidence suggests using albendazole alone or in combination with praziquantel for inoperable patients, patients with multiple cysts, and in $<5-\mathrm{cm}$ CE 1 and CE3a cysts. Mebendazole is considered an alternative drug when the other drugs are not available. ${ }^{4}$ A recent meta-analysis may suggest that the combined treatment with albendazole plus praziquantel is superior to treatment with albendazole alone, with higher scolicidal activity and greater number of cysts cured or improved. ${ }^{11}$ Another key point is the duration of the treatment, where a consensus is not well established because of the weak evidence. This lack of scientific evidence explains the heterogeneity of pharmacological treatment duration observed in our study.

Surgical treatment was the most used therapeutic approach among the participant centers. Although the more radical surgical interventions have higher operative risks, this kind of interventions have showed a lower risk of recurrence $(3.2 \%)$ than more conservative surgical techniques (24\%). ${ }^{12}$ However, surgical treatment (as well as percutaneous techniques) should be combined with pre- and post-pharmacological treatment. ${ }^{10}$ Once again, to date, there are insufficient data to establish the optimal duration of pharmacological treatment when given before and after surgical or percutaneous procedures. High variability was also observed in our study regarding treatment duration in these situations.

Our study has some limitations. Given the nature of the study design (survey-based), a bias could have taken place, more interested and expert CE clinicians could have more chance to answer the survey than those with less experience in the field. However, participants in the study came from centers with very different level of specialization, and the Spanish geographical representation of the centers was wide. Another point to take into account is that some information (number of patients attended, treatment options, and duration of treatment) was collected retrospectively; hence, some information could have been missed. Moreover, the approach depending on the cyst stages was not assessed, and it could be difficult to interpret the results.

In summary, the results of the survey performed among Spanish CE-treating clinicians showed heterogeneity regarding the availability of the different techniques and procedures, as well as in the use of these procedures and recommendations followed. Main differences were found in the PAIR technique use, different drugs administered, duration of pharmacological treatment, and recommendations/ guidelines followed. These results stress the importance of promoting the diffusion of existing knowledge and referring complicated patients to referral centers at a national level. Multidisciplinary teams would decrease not only variability among different centers but also variability within the center. Given the relative low number of patients attended in most of the centers, a national database with clinical and epidemiological information of patients with CE could help homogenize the therapeutic approach of these patients. In this regard, there are initiatives such as the European Register of Cystic Echinococcosis, which Spanish centers could join. ${ }^{13}$

TABLE 3

Comparison of the main characteristics of the centers depending on the number of visited patients in the last three years

\begin{tabular}{|c|c|c|c|}
\hline & Centers attending $\leq 15$ patients $(n=14)$ & Centers attending $>15$ patients $(n=25)$ & $P$-value \\
\hline Access to PAIR & $3 / 14(21.4 \%)$ & $15 / 25(60 \%)$ & 0.020 \\
\hline Serological test available & 12/14 (85.7\%) & $25 / 25(100 \%)$ & 0.123 \\
\hline Median $\%$ of pharmacological treatment & $14.6 \%(0-42.8 \%)$ & $15 \%(4.7-30.3 \%)$ & 0.803 \\
\hline Median $\%$ of surgical treatment & $33.3 \%(19.1-87.5 \%)$ & $46.6 \%$ (34.5-72.9\%) & 0.561 \\
\hline Median $\%$ of PAIR & $0 \%(0-0 \%)$ & $0 \%(0-5.3 \%)$ & 0.325 \\
\hline Median $\%$ of watch and wait & $12.5 \%(0-37.5 \%)$ & $23.8 \%(13.8-33.3 \%)$ & 0.561 \\
\hline Treatment with albendazole & 13/14 (92.8\%) & $24 / 25(96 \%)$ & 1 \\
\hline Combined treatment (albendazole + PZQ) & 4/14 (28.5\%) & $13 / 25(52 \%)$ & 0.157 \\
\hline Median duration of treatment (months) & $3(3-6)$ & $3(3-6)$ & 0.897 \\
\hline $\begin{array}{l}\text { Median duration of pre-IQ treatment } \\
\text { (days) }\end{array}$ & $10.5(6.2-30)$ & $7(7-30)$ & 0.988 \\
\hline $\begin{array}{l}\text { Median duration of post-IQ treatment } \\
\text { (days) }\end{array}$ & $49(29.5-85.5)$ & $30(28-60)$ & 0.251 \\
\hline Suppressive treatment & $5 / 14(35.7 \%)$ & $14 / 25(56 \%)$ & 0.224 \\
\hline Multidisciplinary team & $2 / 14(14.3 \%)$ & $3 / 25(12 \%)$ & 1 \\
\hline Referring patients & 3/14 (21.4\%) & $4 / 25(16 \%)$ & 0.686 \\
\hline Median $\%$ of immigrant patients & $61 \%(12-100 \%)$ & $15 \%(9.5-39.5 \%)$ & 0.045 \\
\hline Median $\%$ of extrahepatic cases & $14 \%(0-33 \%)$ & $15 \%(5-38 \%)$ & 0.284 \\
\hline
\end{tabular}

IQR = interquartile range; PAIR = puncture, aspiration, injection, and reaspiration. Data are reported as number (\%) of centers or median (IQR). 
Received December 23, 2019. Accepted for publication April 1, 2020.

Published online May 11, 2020.

Note: Supplemental material appears at www.ajtmh.org.

Hydatidosis Working Group of the Spanish Society of Tropical Medicine and International Health (SEMTSI): David Carmena, Ma Jesús Perteguer (Centro Nacional Microbiología, Instituto de Salud Carlos III, Madrid), Antonio Muro (Centro de Investigación en Enfermedades Tropicales de la Universidad de Salamanca, CIETUS), Albert Picado (Foundation for Innovative New Diagnostics, FIND), Adrián SánchezMontalvá (Hospital Universitario Vall d'Hebron), José Manuel Ruiz Giardin $(H$. Universitario Fuenlabrada), Cristina Soler $(H$. de Santa Caterina), Ángel Domínguez (H. Universitario Virgen de la Macarena), Juan Carlos García (Complejo Hospitalario Universitario de Pontevedra), Silvia Roure (H. Germans Trias i Pujol), Joaquín Salas-Coronas ( $\mathrm{H}$. de Poniente, El Ejido, Almería), Juan María Herrero, Marta MoraRillo (H. Universitario La Paz-Carlos III IdiPAZ), Jara Llenas-García $(\mathrm{H}$. Vega Baja-FISABIO), Alejandro Salinas (H. de Denia), Gerardo RojoMarcos (H. Príncipe de Asturias), María Arrizabalaga ( $H$. Son Llatzer), Nieves Jaén $(H$. Universitario Insular de Gran Canaria), Yolanda Meije ( $\mathrm{H}$. de Barcelona), Josune Goikoetxea ( $\mathrm{H}$. Universitario Cruces), María Velasco $(H$. Universitario Fundación Alcorcón), Carmen Sáez $(H$. Universitario La Princesa), Eva Ferreira (H. de Segovia), Carlos Dueñas (H. Clínico Universitario de Valladolid), Ángel Chocarro, Felipe Navía ( $\mathrm{H}$. Virgen de la Concha de Zamora), Judit Villar ( $\mathrm{H}$. del Mar), Maite Pérez (Complejo Hospitalario Universitario de Vigo), Pablo Bachiller (H. Universitario Río Hortega), Elías Cañas, Julia Praena (H. Universitario Virgen del Rocío), Patricia Sorni ( $H$. de Manacor), Laura Prieto (H. Fundación Jiménez Díaz), José Luis Mostaza (Complejo Asistencial Universitario de León), Gloria Sampériz (H. Ernest Lluch), Laura Lladó (H. de Bellvitge), José Antonio Díaz (Complejo Hospitalario de Santiago), Ana Pérez-Ayala (H. Universitario 12 de Octubre).

Financial support: This research did not receive any specific grant from funding agencies in the public, commercial, or not-for-profit sectors. This study was supported by the Spanish Society of Tropical Medicine and International Health (SEMTSI) and the ISCIIICollaborative Research Network on Tropical Diseases (RICET), and the European Regional Development Fund (ERDF): RD16/0027/0002, RD16/0027/0003, RD16/0027/0004, RD16/0027/0013, RD16/0027/ 0017, RD16/0027/0018, and RD16/0027/0020.

Authors' addresses: Fernando Salvador, Department of Infectious Diseases, Vall d'Hebron University Hospital, PROSICS Barcelona, Barcelona, Spain, E-mail: fmsalvad@vhebron.net, Eva Calabuig, Infectious Diseases Unit, La Fe University and Polytechnic Hospital, University of Valencia, Spain, E-mail: evacala@yahoo.com.ar. Rogelio López-Vélez, National Referral Unit for Tropical Diseases, Infectious Diseases Department, Ramón y Cajal University Hospital, Madrid, Spain, E-mail: rogelio.lopezvelez@salud.madrid.org. Javier PardoLledías, Department of Internal Medicine, Hospital Universitario Marqués de Valdecilla, Universidad de Cantabria, IDIVAL, Santander, Spain, E-mail: javipard2@hotmail.es. Diego Torrús, Infectious Diseases Unit, Hospital General Universitario de Alicante, Alicante, Spain, E-mail: diemen@coma.es. María Peñaranda, Department of Internal Medicine, Son Espases University Hospital, Palma de Mallorca, Spain, E-mail: maria.penaranda@ssib.es. Azucena Rodríguez-Guardado, Department of Internal Medicine, Hospital Universitario de Cabueñes, Gijón, Spain, E-mail: azucenarodriguez@telecable.es.
Milagros García López-Hortelano, Infectious Diseases Department, La Paz-Carlos III Pediatric University Hospital, Madrid, Spain, E-mail: mghortelano@salud.madrid.org. Moncef Belhassen-García, Department of Internal Medicine, Infectious Diseases Section, Complejo Asistencial Universitario de Salamanca, Salamanca, Spain, E-mail: belhassen@usal.es.

\section{REFERENCES}

1. World Health Organization, 2019. Echinococcosis. Available at: https://www.who.int/echinococcosis/en/. Accessed October 12, 2019.

2. Grosso G, Gruttadauria S, Biondi A, Marventano S, Mistretta A, 2012. Worldwide epidemiology of liver hydatidosis including the Mediterranean area. World J Gastroenterol 18: 1425-1437.

3. Herrador Z, Siles-Lucas M, Aparicio P, López-Vélez R, Gherasim A, Gárate T, Benito A, 2016. Cystic echinococcosis epidemiology in Spain based on hospitalization records, 1997-2012. PLoS Negl Trop Dis 10: e0004942.

4. Brunetti E, Kern P, Vuitton DA; Writing Panel for the WHO-IWGE, 2010. Expert consensus for the diagnosis and treatment of cystic and alveolar echinococcosis in humans. Acta Tropica 114: 1-16.

5. Nabarro LE, Amin Z, Chiodini PL, 2015. Current management of cystic echinococcosis: a survey of specialist practice. Clin Infect Dis 60: 721-728.

6. Romero-Alegría A, Belhassen-García $M$, Alonso-Sardón $M$, Velasco-Tirado V, López-Bernus A, Carpio-Pérez A, Bellido JL, Muro A, Cordero M, Pardo-Lledias J, 2017. Imported cystic echinococcosis in western Spain: a retrospective study. Trans $R$ Soc Trop Med Hyg 110: 664-669.

7. Escolà-Vergé $L$ et al., 2019. Retrospective study of cystic echinococcosis in a recent cohort of a referral center for liver surgery. J Gastrointest Surg 23: 1148-1156.

8. Jiménez S, Pérez A, Gil H, Schantz P, Ramalle E, Juste R, 2002. Progress in control of cystic echinococcosis in La Rioja, Spain: decline in infection prevalences in human and animal hosts and economic costs and benefits. Acta Trop 83: 213-221.

9. Lissandrin R, Vola A, Tahiri S, Mariconti M, Manciulli T, Tamarozzi $F$, Brunetti E, 2018. Cystic echinococcosis in immigrants and Italians accessing a single referral center in Lombardy, Italy. Travel Med Infect Dis [Epub ahead of print].

10. Velasco-Tirado V et al., 2018. Management of cystic echinococcosis in the last two decades: what have we learned? Trans $R$ Soc Trop Med Hyg 112: 207-215.

11. Velasco-Tirado $V$ et al., 2018. Medical treatment of cystic echinococcosis: systematic review and meta-analysis. BMC Infect Dis 18: 306.

12. Aydin U, Yazici P, Onen Z, Ozsoy M, Zeytunlu M, Kiliç M, Coker A, 2008. The optimal treatment of hydatid cyst of the liver: radical surgery with a significant reduced risk of recurrence. Turk $J$ Gastroenterol 19: 33-39.

13. Rossi P, Tamarozzi F, Galati F, Pozio E, Akhan O, Cretu CM, Vutova K, Siles-Lucas M, Brunetti E, Casulli A; HERACLES extended network, 2016. The first meeting of the European register of cystic echinococcosis (ERCE). Parasit Vectors 9: 243. 